\title{
THE INTEGRAL CHOW RING OF THE STACK OF HYPERELLIPTIC CURVES OF EVEN GENUS
}

\author{
Dan Edidin and Damiano Fulghesu
}

\begin{abstract}
Let $g$ be an even positive integer. In this paper we compute the integral Chow ring of the stack of smooth hyperelliptic curves of genus $g$.
\end{abstract}

\section{Introduction}

A natural question, inspired by David Mumford's classic paper Toward an enumerative geometry of the moduli space of curves [13], is to compute the integral Chow rings of the stacks $\mathcal{M}_{g}$ and $\overline{\mathcal{M}}_{g}$ of smooth and stable curves of a given genus $g$. In [6] the integral Chow rings of the stacks of elliptic curves $\mathcal{M}_{1,1}$ and $\overline{\mathcal{M}}_{1,1}$, were computed. In an appendix to the same paper Vistoli [14] computed the Chow ring of $\mathcal{M}_{2}$. However, for $g \geq 3$ almost nothing is known. The only positive result is the computation of $\operatorname{Pic}\left(\mathcal{M}_{g}\right)$ and $\operatorname{Pic}\left(\overline{\mathcal{M}}_{g}\right)$ by Arbarello and Cornalba [1] using Harer's computation of the second homology group of the mapping class group [11]. Even rationally, the Chow rings of $\mathcal{M}_{g}$ have been computed only up to to $g=5[7,8,12]$.

In this paper we turn our attention to hyperelliptic curves and obtain a result valid for all (even) genera. To be precise, let $\mathcal{H}_{g}$ denote the stack of smooth hyperelliptic curves of even genus $g$ defined over a field $k$.

Theorem 1.1. Assume that char $k=0$ or char $k>2 g+1$. Then

$$
A^{*}\left(\mathcal{H}_{g}\right)=\mathbb{Z}\left[c_{1}, c_{2}\right] /\left(2(2 g+1) c_{1}, g(g-1) c_{1}^{2}-4 g(g+1) c_{2}\right) .
$$

Remark 1.2. When $g=2$, every curve is hyperelliptic and our theorem recovers Vistoli's presentation for $A^{*}\left(\mathcal{M}_{2}\right)$. Theorem 1.1 also recovers Arsie and Vistoli's result [2] that $\operatorname{Pic}\left(\mathcal{H}_{g}\right)$ is cyclic of order $2(2 g+1)$.

Remark 1.3. Note that the generators $c_{1}, c_{2}$ of $A^{*}\left(\mathcal{H}_{g}\right)$ are not in general tautological classes. Gorchinskiy and Viviani [10] observed that $\operatorname{Pic}\left(\mathcal{H}_{g}\right)$ is not generated by $\lambda$, the first Chern class of the Hodge bundle, when $g \equiv 0 \bmod 4$. In Section 5.1 we describe a natural vector bundle on $\mathcal{H}_{g}$ whose Chern classes generate the Chow ring.

This theorem is quite surprising since it implies that integral Chow rings of stacks of hyperelliptic curves of even genus have a remarkably simple structure. This is in marked contrast to the situation in topology, where results about the additive structure of the cohomology of the hyperelliptic mapping class group are quite complicated (cf. [4]). In parallel with topology, our theorem implies that $A^{*}\left(\mathcal{H}_{g}\right)$ is all primary $p$-torsion for primes $p \leq 2 g+1$. This confirms a guess made Totaro in his review of Arsie and Vistoli's paper (MR2041774).

Received by the editors January 16, 2008. 
Our techniques are purely algebraic and make essential use of results of Arsie and Vistoli [2]. As observed in [2, Example 3.5] the stack $\mathcal{H}_{g}$ may be identified as the stack of double covers of $\mathbb{P}^{1}$ branched at $2 g+2$ points. Theorem 4.1 of [2] then implies that if $g$ is even $\mathcal{H}_{g}$ is the quotient (stack) by an action of $\mathrm{GL}_{2}$ on the open set in $\mathbb{A}^{2 g+3}$ corresponding to homogeneous binary forms of degree $2 g+2$ with distinct roots. Using a basic result in equivariant intersection theory we then identify $A^{*}\left(\mathcal{H}_{g}\right)$ with the $\mathrm{GL}_{2}$-equivariant Chow ring of this open set.

From this description it immediately follows from the basic exact sequence in Chow groups that $A^{*}\left(\mathcal{H}_{g}\right)$ is a quotient of $A_{\mathrm{GL} 2}^{*} \mathbb{A}^{2 g+3}=\mathbb{Z}\left[c_{1}, c_{2}\right]$. Because inclusion of an open set does not induce a surjection on cohomology, the calculation in topology is much more difficult. Note that this situation is reversed for $\mathcal{M}_{g}$ where there seems to be no algebraic way to get information about the Chow groups for general $g$.

Following a strategy employed by Vistoli in his calculation of $A^{*}\left(\mathcal{M}_{2}\right)$ the computation of the equivariant Chow ring can be reduced to the calculation of the $\mathrm{GL}_{2}{ }^{-}$ equivariant Chow ring of $\mathbb{P}^{2 g+2} \backslash \Delta_{1}$, where $\Delta_{1}$ is the locus of forms divisible by a square. Rather than tackle this problem directly, as Vistoli did for $g=2$, we reduce to the maximal torus $T \subset \mathrm{GL}_{2}$. The technical heart of this paper is the proof that, for any $N, A_{T}^{*}\left(\mathbb{P}\left(\operatorname{Sym}^{N} E^{*}\right) \backslash \Delta_{1}\right)$ is the quotient of $A_{T}^{*}\left(\mathbb{P}\left(\operatorname{Sym}^{N} E^{*}\right)\right)$ by an ideal generated by two classes (Proposition 4.2), where $E$ is the defining representation of $\mathrm{GL}_{2}$. Because $\mathrm{GL}_{2}$ is special ${ }^{1}$ we can then recover the $\mathrm{GL}_{2}$-equivariant Chow ring.

\section{Background on group actions and equivariant intersection theory}

2.1. Group actions and representations. Let $k$ be a field and let $G$ be an algebraic group over $k$. If $V$ is a representation of $G$ over $k$, then, when the context is clear, we refer to the scheme $\operatorname{Spec}\left(\operatorname{Sym} V^{*}\right)$ as $V$. With this convention if $x \in \operatorname{Spec}\left(\operatorname{Sym} V^{*}\right)$ is a $k$-valued point corresponding to a vector $v \in V$ then for any $g \in G(k), g x$ is the $k$-valued point corresponding to the vector $g v$. If the action of $G$ on $V$ commutes with the diagonal action of $\mathbb{G}_{m}$ on $V$ then there is an induced action of $G$ on $\mathbb{P}(V):=\operatorname{Proj}\left(\operatorname{Sym} V^{*}\right)$. As a consequence, if $d$ is positive integer then the global sections $H^{0}\left(\mathbb{P}(V), \mathcal{O}_{\mathbb{P}(V)}(d)\right)$ correspond to the $G$-module $\mathrm{Sym}^{d} V^{*}$.

2.2. Some general facts about equivariant Chow groups. Equivariant Chow groups are defined in the paper [6]. We briefly recall some basic facts and notation that we will use in our computation.

Let $G$ be a linear algebraic group defined over a field $k$. For any algebraic space $X$ we denote the direct sum of the equivariant Chow groups by $A_{*}^{G}(X)$. If $X$ is smooth then there is a product structure on equivariant Chow groups and we denote the equivariant Chow ring by $A_{G}^{*}(X)$. Following standard notation, we denote the equivariant Chow ring of a point by $A_{G}^{*}$. Flat pullback $X \rightarrow$ Spec $k$ makes the equivariant Chow groups $A_{*}^{G}(X)$ into an $A_{G}^{*}$-module. When $X$ is smooth the equivariant Chow ring $A_{G}^{*}(X)$ becomes an $A_{G}^{*}$ algebra.

The relation between equivariant Chow rings and Chow rings of quotient stacks is given by the following result.

Proposition 2.1. [6, Propositions 17, 19] Let $G$ be an algebraic group and let $X$ be a smooth $G$-space and let $\mathcal{F}=[X / G]$ be the quotient stack. Then the equivariant Chow

\footnotetext{
${ }^{1}$ This means that every $\mathrm{GL}_{n}$-torsor is locally trivial in the Zariski topology.
} 
ring $A_{G}^{*}(X)$ is independent of the presentation for $\mathcal{F}$ and may be identified with the integral Chow ring of $\mathcal{F}$.

2.3. Equivariant Chow rings for $\mathrm{GL}_{n}$ actions. Let $T=\mathbb{G}_{\mathbf{m}}^{n}$ be a maximal torus. Because $\mathrm{GL}_{n}$ is special the restriction homomorphism $A_{\mathrm{GL}_{n}}^{*} \rightarrow A_{T}^{*}$ is injective and the image consists of the classes invariant under the action of the Weyl group $W\left(T, \mathrm{GL}_{n}\right)=S_{n}$. Hence, we may view $A_{\mathrm{GL}_{n}}^{*}$ as a sub-algebra of $A_{T}^{*}$. More generally [6, Proposition 3.6] or [3, Theorem 6.7]) imply that if $X$ is an algebraic space then the restriction map $A_{*}^{\mathrm{GL}_{n}}(X) \rightarrow A_{*}^{T}(X)$ is an injective homomorphism of $A_{\mathrm{GL}_{n}}^{*}$-modules. Likewise, if $X$ is smooth, the restriction map $A_{\mathrm{GL}_{n}}^{*} X \rightarrow A_{T}^{*} X$ is an injective homomorphism of $A_{\mathrm{GL}_{n}}^{*}$-algebras. In both cases the images consist of elements which are invariant under the natural action of the Weyl group. If $A_{*}^{\mathrm{GL}_{n}}(X)$ is a flat $A_{\mathrm{GL}}^{*}$ module then [3, Theorem 6.7] also implies ${ }^{2}$ that $A_{*}^{\mathrm{GL}}(X)=\left(A_{*}^{T}(X)\right)^{S_{n}}$.

The following result will be very useful in the proof of Theorem 1.1.

Proposition 2.2. Let $G$ be a special algebraic group and let $T \subset G$ be a maximal torus. If $X$ is a smooth $G$-space then $A_{G}^{*}(X)$ is (non-canonically) a summand in the $A_{G}^{*}(X)$-module $A_{T}^{*}(X)$.

Proof. Fix an integer $i$ and let $V$ be a representation of $G$ which contains an open set $U \subset V$ on which $G$ acts freely such that $V \backslash U$ has codimension $>i$. Then we can identify $A_{G}^{i}(X)=A^{i}\left(X \times_{G} U\right)$ and $A_{T}^{i}(X)=A^{i}\left(X \times_{T} U\right)$. The restriction map $A_{G}^{i}(X) \rightarrow A_{T}^{i}(X)$ corresponds to the flat pullback $\pi: X \times \times_{T} U \rightarrow X \times{ }_{G} U$. Let $B \supset T$ be a Borel subgroup. Since $B / T$ is isomorphic to affine space we may also identify $A_{T}^{i}(X)$ with $A^{i}\left(X \times{ }_{B} U\right)$. Since $G$ is special the $G / B$ bundle $p: X \times_{B} U \rightarrow X \times_{G} U$ is locally trivial in the Zariski topology. Hence by [5, Lemma 7], $A^{*}\left(X \times_{G} U\right)$ is (non-canonically) a summand in $A^{*}\left(X \times{ }_{B} U\right)$.

2.4. Chern classes and equivariant Chow rings of projective spaces. If $V$ is a representation of $G$, then $V$ defines a $G$-equivariant vector bundle over Spec $k$. Consequently a representation $V$ of rank $r$ has Chern classes $c_{1}(V), \ldots, c_{r}(V) \in A_{G}^{*}$. If $X$ is a smooth algebraic space then we will view the Chern classes as elements of the equivariant Chow ring $A_{G}^{*}(X)$ via the pullback $A_{G}^{*} \rightarrow A_{G}^{*}(X)$.

Now let $E$ be the defining representation of $\mathrm{GL}_{n}$. The total character of the $T$ module $E$ decomposes into a sum of linearly independent characters $\lambda_{1}+\lambda_{2}+\ldots+\lambda_{n}$ and we get $A_{T}^{*}=\mathbb{Z}\left[t_{1}, \ldots, t_{n}\right]$ where $t_{i}=c_{1}\left(\lambda_{i}\right)$. The Weyl group $S_{n}$ acts on $A_{T}^{*}$ by permuting the $t_{i}$ 's and as result $A_{\mathrm{GL}_{n}}^{*}=\mathbb{Z}\left[c_{1}, \ldots, c_{n}\right]$ where $c_{i}=c_{i}(E)$ is the $i$-th elementary symmetric polynomial in $t_{1}, \ldots, t_{n}$ [5].

If $V$ is a representation of rank $r$ of $\mathrm{GL}_{n}$ then the total character of the $T$-module $V$ decomposes as sum of characters $\mu_{1}+\ldots+\mu_{r}$. Let $l_{i}=c_{1}\left(\mu_{i}\right)$. We refer to the classes $l_{1}, \ldots, l_{r}$ as the Chern roots of $V$ and view them as elements in $A_{T}^{*} X$. Any symmetric polynomial in the Chern roots is an element of $A_{\mathrm{GL}}^{*} X$.

Let $V$ be an $(r+1)$-dimensional representation of $\mathrm{GL}_{n}$. Since the action of $\mathrm{GL}_{n}$ commutes with the diagonal action of $\mathbb{G}_{m}$ on $V$ there is an induced action of $\mathrm{GL}_{n}$ on $\mathbb{P}(V)$ a canonical $\mathrm{GL}_{n}$-linearization of the sheaf $\mathcal{O}_{\mathbb{P}(V)}(1)$.

\footnotetext{
${ }^{2}$ In [6] it was incorrectly claimed that if $G$ is special and $T$ is a maximal torus then for all $G$-spaces $X, A_{G}^{*}(X)=\left(A_{T}^{*}(X)\right)^{W}$ where $W=W(G, T)$ is the Weyl group. The second author is grateful to Reyer Sjamaar for pointing out this error.
} 
The following easy lemma is proved for torus actions in [6, Section 3.3] and follows in general from the projective bundle theorem [9, Example 8.3.4].

Lemma 2.3. The $\mathrm{GL}_{n}$ (resp. $T$ ) equivariant Chow ring of $\mathbb{P}(V)$ has the following presentation.

$$
A_{\mathrm{GL}_{n}}^{*}(\mathbb{P}(V))=A_{\mathrm{GL}_{n}}^{*}[\xi] /\left(\xi^{r+1}+C_{1} \xi^{n}+\ldots+C_{r+1}\right)
$$

and

$$
A_{T}^{*}(\mathbb{P}(V))=A_{T}^{*}[\xi] /\left(\prod_{i=1}^{r}\left(\xi+l_{i}\right)\right)
$$

where $\xi=c_{1}\left(\mathcal{O}_{\mathbb{P}(V)}(1)\right)$ and $C_{1}, \ldots, C_{r} \in A_{\mathrm{GL}_{n}}^{*}$ (resp. $\left.l_{1}, \ldots, l_{r}\right)$ are the equivariant Chern classes (resp. Chern roots) of the representation $V$.

2.5. T-equivariant fundamental classes of invariant hypersurfaces. Let $T$ be a torus and let $V$ be a finite dimensional $T$-module and let $\mathcal{O}_{\mathbb{P}(V)}(1)$ have the canonical linearization induced by the action of $T$ on $V$.

Lemma 2.4. Let $H \subset \mathbb{P}(V)$ be a $T$-invariant hypersurface defined by a homogeneous form $f \in \operatorname{Sym}^{d}\left(V^{*}\right)$ such that $z \cdot f=\chi^{-1}(z) f$ for some character $\chi: T \rightarrow \mathbb{G}_{m}$. Then in $A_{T}^{*}(\mathbb{P}(V))$,

$$
[H]_{T}=c_{1}^{T}\left(\mathcal{O}_{\mathbb{P}(V)}(d)\right)+c_{1}(\chi)
$$

Proof. On the invariant affine $a_{i} \neq 0$, the ideal sheaf of $H$ is generated by the $T$ eigenfunction $f / x_{i}$. Hence $\mathcal{O}(-H)=\mathcal{O}(-d) \otimes \chi^{-1}$ where $\mathcal{O}(-d)$ is given its canonical $T$-linearization.

Let $V$ be a two-dimensional representation of $T$ and choose coordinates so that $T$ acts by $z \cdot\left(a_{0}, a_{1}\right)=\left(\chi_{0}(z) a_{0}, \chi_{1}(z) a_{1}\right)$. Let $\mathbb{P}^{1}=\mathbb{P}(V)$ and let $\Delta \subset \mathbb{P}^{1} \times \mathbb{P}^{1}$ be the diagonal. Then $\Delta$ is a $T$-invariant hypersurface for the diagonal action of $T$ on $\mathbb{P}^{1} \times \mathbb{P}^{1}$.

Lemma 2.5. In $A_{T}^{*}\left(\mathbb{P}^{1} \times \mathbb{P}^{1}\right)$ we have the identity

$$
\left[\Delta_{\mathbb{P}^{1} \times \mathbb{P}^{1}}\right]=\left[0 \times \mathbb{P}^{1}\right]+\left[\mathbb{P}^{1} \times 0\right]+c_{1}\left(\chi_{1} \chi_{0}^{-1}\right)
$$

Proof. Let $x_{0}, x_{1}$ (resp. $\left.y_{0}, y_{1}\right)$ be coordinate functions on the first (resp. second) copy of $\mathbb{P}^{1}$. Then $\Delta_{\mathbb{P}^{1} \times \mathbb{P}^{1}}$ is defined by the homogeneous quadratic form $x_{0} y_{1}-x_{1} y_{0}$ while $\left[0 \times \mathbb{P}^{1}\right]+\left[\mathbb{P}^{1} \times 0\right]$ is defined by the homogeneous quadratic form $x_{0} y_{0}$. Now $x_{0} y_{1}-x_{1} y_{0}$ is in the $\chi_{0}^{-1} \chi_{1}^{-1}$ eigenspace of $V^{*} \otimes V^{*}$ and $x_{0} y_{0}$ while is in the $\chi_{0}^{-2}$ eigenspace. The formula now follows from the same argument used in Lemma 2.4.

\section{Arsie and Vistoli's presentation $\mathcal{H}_{g}$ as quotient stack}

To reduce the computation of the $A^{*}\left(\mathcal{H}_{g}\right)$ to a calculation in equivariant intersection theory we recall the presentation for $\mathcal{H}_{g}$ for $g$ even given by Arsie and Vistoli in $[2]$.

Let $k$ be a field of characteristic not equal 2 and assume that $g$ is an even integer. Let $E$ be the defining representation of $\mathrm{GL}_{2}$ and let $\mathcal{D}: \mathrm{GL}_{2} \rightarrow \mathbb{G}_{m}$ be the determinant. 
Theorem 3.1. [2, Corollary 4.7] If $g$ is even the stack $\mathcal{H}_{g}$ is isomorphic to the quotient

$$
\left[\left(\mathrm{Sym}^{2 g+2} E^{*} \otimes \mathcal{D}^{\otimes g} \backslash \Delta_{1}\right) / \mathrm{GL}_{2}\right]
$$

where $\Delta_{1}$ is the closed subvariety of singular forms.

By Proposition 2.1 the Chow ring of $\mathcal{H}_{g}$ may identified with the equivariant Chow ring

$$
A_{\mathrm{GL}}^{*}\left(\mathrm{Sym}^{2 g+2} E^{*} \otimes \mathcal{D} \backslash \Delta_{1}\right) .
$$

and the remainder of the paper is devoted to performing this computation.

Let $\mathbb{P}^{2 g+2}=\mathbb{P}\left(\mathrm{Sym}^{2 g+2} E^{*}\right)$ be the projective space of forms of degree $2 g+2$ and again let $\Delta_{1}$ be the hypersurface corresponding to singular forms. Following the argument of Vistoli [14] we may reduce to a calculation in $\mathbb{P}^{2 g+2}$.

Lemma 3.2. Let $\xi$ denote the first Chern class of $\mathcal{O}_{\mathbb{P}^{2 g+2}}(1)$. Then the pull-back

$$
A_{\mathrm{GL}}^{*}\left(\mathbb{P}^{2 g+2} \backslash \Delta_{1}\right) \stackrel{\Pi^{*}}{\longrightarrow} A_{\mathrm{GL}_{2}}^{*}\left(\mathrm{Sym}^{2 g+2} E \otimes \mathcal{D}^{\otimes g} \backslash \Delta_{1}\right)
$$

is surjective and its kernel is generated by $g c_{1}-\xi$.

Proof. In general if $\Pi: X \rightarrow Y$ is a $G$-equivariant $\mathbb{G}_{\mathbf{m}}$-bundle we consider the associated line bundle $\mathcal{L}$. Then $X$ is the complement of the 0 -section and the localization exact sequence for equivariant Chow groups implies that

$$
A_{G}^{*}(Y) / c_{1}(\mathcal{L}) \cong A_{G}^{*}(X)
$$

where the isomorphism is induced by $\Pi^{*}$. In our case the associated line bundle is $\mathcal{D}^{\otimes g} \otimes \mathcal{O}(-1)$ so the kernel of $\Pi^{*}$ is $g c_{1}-t$.

From Lemma 3.2 we conclude that

$$
A^{*} \mathcal{H}_{g}=A_{\mathrm{GL}_{2}}^{*}\left(\mathbb{P}^{2 g+2} \backslash \Delta_{1}\right) /\left(g c_{1}-\xi\right)
$$

so we have reduced the problem to computing

$$
A_{\mathrm{GL}_{2}}^{*}\left(\mathbb{P}^{2 g+2} \backslash \Delta_{1}\right)=A_{\mathrm{GL}_{2}}^{*}\left(\mathbb{P}^{2 g+2}\right) / I
$$

where $I$ is the ideal generated by the image of $A_{*}^{\mathrm{GL}_{2}}\left(\Delta_{1}\right)$.

\section{The equivariant Chow ring of the space of non-degenerate homogeneous forms in $N$ variables}

Let $E$ be the defining representation of $\mathrm{GL}_{2}$ and let $\mathbb{P}^{N}=\mathbb{P}\left(\mathrm{Sym}^{N} E^{*}\right)$ be the projective space of homogeneous forms of degree $N$ in two variables $x_{0}$ and $x_{1}$. Since the action of $\mathrm{GL}_{2}$ commutes with homotheties, there is an induced action of $\mathrm{GL}_{2}$ on $\mathbb{P}^{N}$ with kernel the center of $\mathrm{GL}_{2}$.

Let $\Delta_{1} \subset \mathbb{P}^{N}$ be the locus of forms which are divisible by a square over some extension of the base field. This subvariety is $\mathrm{GL}_{2}$ invariant and the goal of this section is to compute the equivariant Chow ring $A_{\mathrm{GL}_{2}}^{*}\left(\mathbb{P}^{N} \backslash \Delta_{1}\right)=A_{\mathrm{GL}_{2}}^{*}\left(\mathbb{P}^{N}\right) / I$ where $I$ is the ideal in $A_{\mathrm{GL}_{2}}^{*}\left(\mathbb{P}^{N}\right)$ generated by the image of the equivariant Chow groups of $\Delta_{1}$. We can then apply the results of this section when $N=2 g+2$ to complete the computation of the integral Chow ring of $\mathcal{H}_{g}$ for $g$ even. (Note, however, that in this section we do not require $N$ to be even.) 
For every $r=1, \ldots,[N / 2]$ we may define $\Delta_{r} \subset \operatorname{Sym}^{N} E^{*}$ as the closed subvariety of forms divisible by the square of a polynomial of degree $r$ over some extension of the ground field $k$. The locus $\Delta_{r}$ is the image of the map

$$
\begin{aligned}
\pi_{r}: \operatorname{Sym}^{r} E^{*} \times \operatorname{Sym}^{N-2 r} E^{*} & \rightarrow \operatorname{Sym}^{N} E^{*} \\
(f, g) & \mapsto f^{2} g
\end{aligned}
$$

Passing to the associated projective spaces we obtain $\mathrm{GL}_{2}$-equivariant maps

$$
\pi_{r}: \mathbb{P}^{r} \times \mathbb{P}^{N-2 r} \rightarrow \mathbb{P}^{N}
$$

and $\Delta_{r}$ will still indicate the image of $\pi_{r}$.

Proposition 4.1. (cf. [14, Lemma 3.3]) If char $k=0$ or char $k>N$ then the image of $A_{*}^{\mathrm{GL}_{2}}\left(\Delta_{1}\right)$ in $A_{\mathrm{GL}_{2}}^{*}\left(\mathbb{P}^{N}\right)$ is the sum of the images of the homomorphisms

$$
\pi_{r *}: A_{*}^{\mathrm{GL}}\left(\mathbb{P}^{r} \times \mathbb{P}^{N-2 r}\right) \rightarrow A_{\mathrm{GL}_{2}}^{*}\left(\mathbb{P}^{N}\right)
$$

Proof. Let $G$ be an algebraic group. Recall [6] that if $X$ is a $G$-scheme then an equivariant envelope is a proper $G$-equivariant morphism $f: \tilde{X} \rightarrow X$ such that for every $G$-invariant subvariety $W \subset X$ there is a $G$-invariant subvariety $\tilde{W} \subset \tilde{X}$ mapping birationally to $X$. Lemma 3 of [6] together with [9, Lemma 18.3(6)] implies that proper pushforward $f_{*}: A_{*}^{G}(\tilde{X}) \rightarrow A_{*}^{G}(X)$ is surjective.

Let

$$
\pi: \coprod_{r=1}^{N / 2} \mathbb{P}^{r} \times \mathbb{P}^{N-2 r} \rightarrow \Delta_{1}
$$

be the $\mathrm{GL}_{2}$-equivariant map whose restriction to $\mathbb{P}^{r} \times \mathbb{P}^{N-2 r}$ is $\pi_{r}$. By the above discussion it suffices to prove that $\pi$ is an equivariant envelope. Let $K \supset k$ be a field. The argument used in the proof [14, Lemma 3.2] shows that, when $r<N / 2$, every $K$-valued point point of $\Delta_{r}$ is the image of unique $K$-valued point of $\mathbb{P}^{r} \times \mathbb{P}^{N-2 r}$ provided char $k=0$ or char $k>2 r+1$. Likewise, if $r=N / 2$ (so $N$ must be even) then there is a unique $K$-valued point of $\mathbb{P}^{N / 2}$ mapping to a $K$-valued point of $\Delta_{N / 2}$ provided char $k=0$ or char $k>N$.

Let $Z \subset \Delta_{1}$ be a $G$-invariant subvariety and suppose that the generic point $\underset{\sim}{p}$ of $Z$ lies in $\Delta_{r} \backslash \Delta_{r+1}$. By Vistoli's Lemma we know that there is subvariety $\tilde{Z} \subset$ $\mathbb{P}^{r} \times \mathbb{P}^{N-2 r}$ mapping birationally to $Z$. To complete the proof we must show that we may take $\tilde{Z}$ to be $G$-invariant. Now if $g \in \mathrm{GL}_{2}$ then the subvariety $g \tilde{Z}$ also maps birationally to $V$. Since there is a unique point of $\mathbb{P}^{r} \times \mathbb{P}^{N-2 r}$ mapping to the generic point $q$, it follows that $g \tilde{Z}$ and $\tilde{Z}$ have the same generic point. Hence $\tilde{Z}$ must contain a $G$-invariant open set $\tilde{U}$. Taking the closure of $\tilde{U}$ in $\mathbb{P}^{r} \times \mathbb{P}^{N-2 r}$ gives our desired $G$-invariant subvariety mapping birationally to $Z$.

For any $r$ let $\xi_{r, 1}$ (respectively $\xi_{N-2 r, 2}$ ) be the pullback to $\mathbb{P}^{r} \times \mathbb{P}^{N-2 r}$ of $c_{1}^{\mathrm{GL}_{2}}(\mathcal{O}(1)$ ) on the first (resp. second) factor. We have the following relation in $A_{\mathrm{GL}_{2}}^{*}\left(\mathbb{P}^{r} \times \mathbb{P}^{N-2 r}\right)$ :

$$
\pi_{r}^{*}(\xi)=2 \xi_{r, 1}+\xi_{N-2 r, 2} .
$$

Moreover, from Lemma 2.3 we have that $\xi_{r, 1}^{r+1}$ is a zero of a monic polynomial of degree $r+1$ with coefficients in $A_{\mathrm{GL}_{2}}^{*}$. Therefore $\pi_{r *}\left(A_{\mathrm{GL}_{2}}^{*}\left(\mathbb{P}^{r} \times \mathbb{P}^{N-2 r}\right)\right)$ is generated as a $A_{\mathrm{GL}_{2}}^{*}\left(\mathbb{P}^{N}\right)$-module by $\pi_{r *}(1), \pi_{r *}\left(\xi_{r, 1}\right), \ldots, \pi_{r *}\left(\xi_{r, 1}^{r}\right)$. 
For $r=1, \ldots,[N / 2]$ and $i=0, \ldots, r$ set

$$
\alpha_{r, i}:=\pi_{r *}\left(\xi_{r, 1}^{i}\right) \text {. }
$$

The above discussion allows us to conclude that

$$
A_{\mathrm{GL}_{2}}^{*}\left(\mathbb{P}^{N} \backslash \Delta_{1}\right)=A_{\mathrm{GL}_{2}}^{*}\left(\mathbb{P}^{N}\right) /\left(\left\{\alpha_{r, i}\right\}\right)
$$

4.1. The ideal generated by $A_{T}^{*}\left(\Delta_{1}\right)$ in $A_{T}^{*}\left(\mathbb{P}^{N}\right)$. The action of $\mathrm{GL}_{2}$ on $\mathbb{P}^{N}$ restricts to an action of the maximal torus $T \subset \mathrm{GL}_{2}$ consisting of diagonal matrices. The goal of this section is to prove

Proposition 4.2. If char $k=0$ or char $k>N$ then the image of $A_{*}^{T}\left(\Delta_{1}\right)$ in $A_{T}^{*}\left(\mathbb{P}^{N}\right)$ is the ideal $\left(\alpha_{1,0}, \alpha_{1,1}\right)$.

(Here we use the notation $\alpha_{r, i}$ to indicate the restriction of the same named classes to $A_{T}^{*}\left(\mathbb{P}^{N}\right)$.)

The proof will require the introduction of alternate, but less symmetric classes which generate the same ideal. The group $T$ acts on $\mathbb{P}^{1}$ by

$$
z \cdot(a: b)=\left(\lambda_{1}^{-1}(z) a: \lambda_{2}^{-1}(z) b\right) .
$$

Choose coordinates $\left(X_{0}: X_{1}: \ldots: X_{N}\right)$ on $\mathbb{P}^{N}$ so that the coordinate function $X_{i}$ is the coefficient of $x_{0}^{N-i} x_{1}^{i}$ in a homogeneous form of degree $N$. Then $T$ acts on $\mathbb{P}^{N}$ by the rule

$$
z \cdot\left(X_{0}: \ldots: X_{i}: \ldots: X_{N}\right)=\left(\lambda_{1}^{-N}(z) X_{0}: \ldots: \lambda_{1}^{i-N} \lambda_{2}^{-i}(z) X_{i}: \ldots: \lambda_{2}^{-N}(z) X_{N}\right) .
$$

Let $H_{i} \subset \mathbb{P}^{N}$ be the hyperplane defined by the equation $X_{i}=0$; in other words, $H_{i}$ corresponds to forms $f$ such that the coefficient of $x_{0}^{N-i} x_{1}^{i}$ is 0 . Let $h_{i}$ be the $T$-equivariant fundamental class of $H_{i}$.

By Lemma 2.4, we have that

$$
\begin{aligned}
h_{i} & =c_{1}(\mathcal{O}(1))+c_{1}\left(\lambda_{1}^{i-N} \lambda_{2}^{-i}\right) \\
& =\xi-(N-i) t_{1}-i t_{2}
\end{aligned}
$$

On $\mathbb{P}^{r}$ we can consider the hyperplane class $h_{r, i}$ corresponding to degree $r$ forms such that the coefficient of $x_{0}^{r-i} x_{1}^{i}$ is 0 . Again by Lemma 2.4 we have that $h_{r, i}=\xi_{r, 1}-$ $(r-i) t_{1}-i t_{2}$. It follows that the image of $A_{*}^{T}\left(\Delta_{1}\right)$ is generated by the pushforwards to $A_{T}^{*}\left(\mathbb{P}^{N}\right)$ of the classes $\pi_{r *}\left(h_{r, 0} \ldots h_{r, m}\right)$ for $1 \leq r \leq[N / 2], 0 \leq m \leq r-1$ as well as the classes $\alpha_{r, 0}$.

Lemma 4.3. In $A_{T}^{*}\left(\mathbb{P}^{N}\right)$ we have the relations

$$
\begin{aligned}
& \alpha_{1,0}=2(N-1) h_{0}+N(N-1)\left(t_{1}-t_{2}\right) \\
& =2(N-1) \xi-N(N-1) c_{1} \\
& \alpha_{1,1}=h_{0} h_{1}+t_{1} \alpha_{1,0} \\
& =\xi^{2}-c_{1} \xi-N(N-2) c_{2}
\end{aligned}
$$

(Recall that $c_{1}=t_{1}+t_{2}$ and $c_{2}=t_{1} t_{2}$ are the elementary symmetric polynomials in the generators $t_{1}, t_{2}$ for $A_{T}^{*}$.)

Remark 4.4. Note that the identities $\alpha_{1,0}=2(N-1) \xi-N(N-1) c_{1}$ and $\alpha_{1,1}=$ $\xi^{2}-c_{1} \xi-N(N-2) c_{2}$ also hold in $A_{\mathrm{GL}_{2}}^{*}\left(\mathbb{P}^{N}\right)$ since the restriction map $A_{\mathrm{GL}_{2}}^{*}\left(\mathbb{P}^{N}\right) \rightarrow$ $A_{T}^{*}\left(\mathbb{P}^{N}\right)$ is injective. 
Proof. The multiplication map $\rho:\left(\mathbb{P}^{1}\right)^{N} \rightarrow \mathbb{P}^{N}$ is $\mathrm{GL}_{2}$-equivariant and hence $T$ equivariant. It also commutes with the natural permutation action of $S_{N}$ on $\left(\mathbb{P}^{1}\right)^{N}$. With this notation, the subvariety $\Delta_{1}$ corresponding to homogeneous forms with multiple roots is the image of $\Delta_{\mathbb{P}^{1} \times \mathbb{P}^{1}} \times\left(\mathbb{P}^{1}\right)^{N-2}$.

Consider the diagram

$$
\begin{array}{cccc}
\left(\Delta_{\mathbb{P}^{1} \times \mathbb{P}^{1}}\right) \times\left(\mathbb{P}^{1}\right)^{N-2} & \hookrightarrow & \left(\mathbb{P}^{1}\right)^{N} \\
\downarrow \rho_{1} & & \downarrow \rho \\
\Delta_{1} & \stackrel{i_{1}}{\hookrightarrow} & \mathbb{P}^{N}
\end{array}
$$

where $\rho$ is the multiplication map and $\rho_{1}$ is its restriction to $\Delta_{\mathbb{P}^{1} \times \mathbb{P}^{1}} \times\left(\mathbb{P}^{1}\right)^{N-2}$. Since $\rho_{1}$ has degree $(N-2)$ ! we see that

$$
(N-2) ! \alpha_{1,0}=\rho_{*}\left[\Delta_{\mathbb{P}^{1} \times \mathbb{P}^{1}} \times\left(\mathbb{P}^{1}\right)^{N-2}\right]
$$

The torus $T$ acts on $\mathbb{P}^{1}$ by $t\left(x_{0}: x_{1}\right)=\left(\lambda_{1}^{-1} x_{0}: \lambda_{2}^{-1} x_{1}\right)$ so by Lemma 2.5

$$
\left[\Delta_{\mathbb{P}^{1} \times \mathbb{P}^{1}}\right]=\left[0 \times \mathbb{P}^{1}\right] \times\left[0 \times \mathbb{P}^{1}\right]+\left(t_{1}-t_{2}\right)\left[\mathbb{P}^{1} \times \mathbb{P}^{1}\right] .
$$

Substituting the right hand side of (8) into the right hand side of (7) we obtain

(9) $(N-2) ! \alpha_{1,0}=\rho_{*}\left(\left[0 \times \mathbb{P}^{1} \times\left(\mathbb{P}^{1}\right)^{N-1}\right]+\left[\mathbb{P}^{1} \times 0 \times\left(\mathbb{P}^{1}\right)^{N-1}\right]+\left(t_{1}-t_{2}\right)\left[\left(\mathbb{P}^{1}\right)^{N}\right]\right)$

The first two terms on the right hand side of (9) pushforward to $(N-1) ! h_{0}$ since the map $0 \times\left(\mathbb{P}^{1}\right)^{N-1} \rightarrow H_{0}$ has degree $(N-1)$ ! while the direct image of the second term is $N !\left(t_{1}-t_{2}\right)$. Thus we obtain the relation

$$
(N-2) ! \alpha_{1,0}=2(N-1) ! h_{0}+N !\left(t_{1}-t_{2}\right)
$$

Since $A_{T}^{*}\left(\mathbb{P}^{N}\right)$ is torsion free we can divide $(10)$ by $(N-2)$ ! to obtain the first identity in (4) and substituting $h_{0}=\xi-N t_{1}$ yields the second.

As noted above we have $h_{1,0}=\xi_{1,1}-t_{1}$ in $A_{T}^{*}\left(\mathbb{P}^{1}\right)$. Thus $\alpha_{1,1}=\pi_{1 *} h_{1,0}+t_{1}\left[\Delta_{1}\right]$. Now $(N-2) ! h_{1,0}=\rho_{1 *}\left(\left[0 \times 0 \times \mathbb{P}^{N-2}\right]\right)$. Thus, after pushing forward to $\mathbb{P}^{N}$ we obtain the identity

$$
\begin{aligned}
(N-2) ! i_{1 *} \pi_{1 *} h_{1,0} & =(N-2) ! \rho_{*}\left[0 \times 0 \times\left(\mathbb{P}^{1}\right)^{N-2}\right] \\
& =(N-2) ! h_{0} h_{1}
\end{aligned}
$$

Since $i_{1 *}\left[\Delta_{1}\right]=\alpha_{1,0}$ the first identity in (5) follows. Substituting $h_{0}=\xi-N t_{1}$ and $h_{1}=\xi+(1-N) t_{1}-t_{2}$ yields the second.

As an immediate consequence of the identities in Lemma 4.3 we obtain.

Lemma 4.5. $\left(\alpha_{1,0}, \alpha_{1,1}\right)=\left(\alpha_{1,0}, h_{0} h_{1}\right)$ as ideals in $A_{T}^{*}\left(\mathbb{P}^{N}\right)$.

If $m \geq 0$ let $\beta_{r, m}$ be the image of the class $h_{r, 0} \ldots h_{r, m}$ in $A_{T}^{*}\left(\mathbb{P}^{r}\right)$.

Lemma 4.6. The class $\beta_{r, m}$ is a multiple of $h_{0} h_{1}$.

Proof. Consider the diagram analogous to (6)

$$
\begin{array}{ccc}
\left(\Delta_{\mathbb{P}^{1} \times \mathbb{P}^{1}}\right)^{r} \times\left(\mathbb{P}^{1}\right)^{N-2 r} & \hookrightarrow & \left(\mathbb{P}^{1}\right)^{N} \\
\downarrow \rho_{r} & & \downarrow \rho \\
\Delta_{r} & \stackrel{i_{r}}{\hookrightarrow} & \mathbb{P}^{N}
\end{array}
$$


Identifying $\Delta_{\mathbb{P}^{1} \times \mathbb{P}^{1}}$ with $\mathbb{P}^{1}$ then $h_{r, 0} \ldots h_{r, m}$ is the equivariant fundamental class of the image of the $T$-invariant subvariety

$$
(0 \times 0) \times \ldots \times(0 \times 0) \times\left(\Delta_{\mathbb{P}^{1} \times \mathbb{P}^{1}}\right)^{r-(m+1)} \times\left(\mathbb{P}^{1}\right)^{N-2 r} .
$$

(Here the first $(2 m+2)$ coordinates are 0$)$. Let $\theta_{r, m}$ be the equivariant fundamental class of this $T$-invariant subvariety Thus, $\rho_{r *} \theta_{r, m}=(N-2 r) !(r-m-1) ! h_{r, 0} \ldots h_{r, m}$. On the other hand we may expand $\theta_{r, m}$ by replacing $\Delta_{\mathbb{P}^{1} \times \mathbb{P}^{1}}$ with the formula of (8) to obtain a sum of classes which are permutations of classes of the form

$$
\left(t_{1}-t_{2}\right)^{m+r+1-l}\left[0 \times 0 \times \ldots \times 0 \times\left(\mathbb{P}^{1}\right)^{N-l}\right]
$$

where first $l$ coordinates are 0 and $2 m+2 \leq l \leq r+m+1$. The action of $T$ on $\left(\mathbb{P}^{1}\right)^{N}$ commutes with natural permutation action of $S_{N}$ and the map $\rho$ is $S_{N^{-}}$-equivariant the pushforward $\rho_{*}$ of the classes in (12) we obtain the identity

$$
(r-(m+1)) !(N-2 r) ! \beta_{r, m}=\sum_{l=2 m+2}^{m+r+1} a_{l}(N-l) !\left(t_{1}-t_{2}\right)^{m+r+1-l} h_{0} \ldots h_{l}
$$

where the $a_{l}$ 's are positive integers. Since $l \leq m+r+1$ it follows that $(r-(m+1)) !(N-$ $2 r)$ ! divides $(N-l)$ !. (This follows because $a !(N-b)$ ! always divides $(N-b+a)$ ! since $\frac{(N-b+a) !}{a !(N-b) !}$ is a binomial coefficient, so $a !(N-b)$ ! divides $(N-k)$ ! if $k \leq b-a$.) Thus, since $A_{T}^{*}\left(\mathbb{P}^{N}\right)$ is torsion free $\alpha_{r, m}$ is an integral sum of terms of the form $\left(t_{1}-t_{2}\right)^{m+r+1-l} h_{0} \ldots h_{l}$ which is clearly a multiple of $h_{0} h_{1}$.

Lemma 4.7. $\alpha_{2,0}$ is in the ideal $\left(\alpha_{1,0}, h_{0} h_{1}\right)$.

Proof. Since $\Delta_{2}$ is the image of the fundamental class of $\Delta_{\mathbb{P}^{1} \times \mathbb{P}^{1}} \times \Delta_{\mathbb{P}^{1} \times \mathbb{P}^{1}} \times\left(\mathbb{P}^{1}\right)^{N-4}$ we see that

$$
2 !(N-4) ! \alpha_{2,0}=\rho_{*}\left[\Delta_{\mathbb{P}^{1} \times \mathbb{P}^{1}} \times \Delta_{\mathbb{P}^{1} \times \mathbb{P}^{1}} \times\left(\mathbb{P}^{1}\right)^{N-4}\right]
$$

Expand the first diagonal term as

$$
\left[\mathbb{P}^{1} \times 0\right]+\left[0 \times \mathbb{P}^{1}\right]+\left(t_{1}-t_{2}\right)\left[\mathbb{P}^{1} \times \mathbb{P}^{1}\right]
$$

and substitute this into the right-hand-side of equation (14) to obtain

$$
\begin{aligned}
2 !(N-4) ! \alpha_{2,0}=\rho_{*} & {\left[0 \times \mathbb{P}^{1} \times \Delta_{\mathbb{P}^{1} \times \mathbb{P}^{1}} \times\left(\mathbb{P}^{1}\right)^{N-4}\right] } \\
& +\rho_{*}\left[\mathbb{P}^{1} \times 0 \times \Delta_{\mathbb{P}^{1} \times \mathbb{P}^{1}} \times\left(\mathbb{P}^{1}\right)^{N-4}\right]+(N-2) !\left(t_{1}-t_{2}\right) \alpha_{1,0}
\end{aligned}
$$

Since the action of $T$ on $\left(\mathbb{P}^{1}\right)^{N}$ commutes with the permutation action of $S_{N}$ the first two terms in the right-hand side of (15) are equal. Now expand the remaining $\Delta_{\mathbb{P}^{1} \times \mathbb{P}^{1}}$ as above so that the sum of the first two terms on the right hand side of (15) now becomes

$$
\begin{aligned}
2\left(\rho_{*}\left[\mathbb{P}^{1} \times 0 \times \mathbb{P}^{1} \times 0 \times\left(\mathbb{P}^{1}\right)^{N-4}\right]\right. & +\rho_{*}\left[\mathbb{P}^{1} \times 0 \times 0 \times \mathbb{P}^{1} \times\left(\mathbb{P}^{1}\right)^{N-4}\right] \\
& \left.+\left(t_{1}-t_{2}\right) \rho_{*}\left[\mathbb{P}^{1} \times 0 \times \mathbb{P}^{1} \times \mathbb{P}^{1} \times\left(\mathbb{P}^{1}\right)^{N-4}\right]\right)
\end{aligned}
$$

Using the fact $\rho_{*}$ commutes with the permutation action of $S_{N}$ we can combine the terms coming from (16) with the last term on the right side of equation (15) to obtain

$$
2 !(N-4) ! \alpha_{2,0}=4(N-2) ! h_{0} h_{1}+2(N-1) !\left(t_{1}-t_{2}\right) h_{0}+(N-2) !\left(t_{1}-t_{2}\right) \alpha_{1,0}
$$


Dividing through by 2 ! $(N-4)$ ! and again invoking the fact that $A_{T}^{*}\left(\mathbb{P}^{N}\right)$ is torsion free we obtain the equation

$$
\begin{aligned}
\alpha_{2,0}=2(N-2)(N-3) h_{0} h_{1}+(N-1)(N-2) & (N-3)\left(t_{1}-t_{2}\right) h_{0} \\
& +\frac{(N-2)(N-3)}{2}\left(t_{1}-t_{2}\right) \alpha_{1,0}
\end{aligned}
$$

The first and third terms on the right-hand-side are clearly in the ideal $\left(\alpha_{1,0}, h_{0} h_{1}\right)$. The middle term is in the ideal because by (3) and (4)

$$
(N-1)(N-2) h_{0}\left(t_{1}-t_{2}\right)=h_{0} \alpha_{1,0}-2(N-1) h_{0} h_{1}
$$

Our last lemma completes the proof of the proposition.

Lemma 4.8. If $r \geq 3$ then $\alpha_{r, 0}$ is in the ideal $\left(\alpha_{1,0}, h_{0} h_{1}\right)$.

Proof. As above we have

$$
r !(N-2 r) ! \alpha_{r, 0}=\rho_{*}\left[\left(\Delta_{\mathbb{P}^{1} \times \mathbb{P}^{1}}\right)^{r} \times\left(\mathbb{P}^{1}\right)^{N-2 r}\right]
$$

Expanding out the first $(r-2)$ copies of the fundamental class of $\Delta_{\mathbb{P}^{1} \times \mathbb{P}^{1}}$ in the right-hand side of $(20)$ we obtain that $\left(\Delta_{\mathbb{P}^{1} \times \mathbb{P}^{1}}\right)^{r} \times\left(\mathbb{P}^{1}\right)^{N-2 r}$ is the sum of

$$
\left(t_{1}-t_{2}\right)^{r-2}\left[\left(\Delta_{\mathbb{P}^{1} \times \mathbb{P}^{1}}\right)^{2} \times\left(\mathbb{P}^{1}\right)^{N-4}\right]
$$

plus terms which are permutations of the $(r-2)$ classes

$$
\left(t_{1}-t_{2}\right)^{r-2-k}\left[\left(0 \times \mathbb{P}^{1}\right)^{k} \times\left(\Delta_{\mathbb{P}^{1} \times \mathbb{P}^{1}}\right)^{2}\right] \times\left(\mathbb{P}^{1}\right)^{N-2(k+2)}
$$

where $1 \leq k \leq r-2$. Since

$$
\rho_{*}\left[\left(\Delta_{\mathbb{P}^{1} \times \mathbb{P}^{1}}\right)^{2} \times\left(\mathbb{P}^{1}\right)^{N-4}\right]=2(N-4) ! \alpha_{2,0}
$$

there are positive integers $b_{1}, \ldots b_{r-2}$ such that

$$
\begin{aligned}
& r !(N-2 r) ! \alpha_{r, 0}=2(N-4) !\left(t_{1}-t_{2}\right)^{r-2} \alpha_{2,0} \\
& +\sum_{k=1}^{r-2} b_{k}\left(t_{1}-t_{2}\right)^{r-2-k} \rho_{*}\left[\left(0 \times \mathbb{P}^{1}\right)^{k} \times\left(\Delta_{\mathbb{P}^{1} \times \mathbb{P}^{1}}\right)^{2} \times\left(\mathbb{P}^{1}\right)^{N-2(k+2)}\right]
\end{aligned}
$$

Now expand $\left[\left(\Delta_{\mathbb{P}^{1} \times \mathbb{P}^{1}}\right)^{2}\right]$ in $A_{T}^{*}\left(\left(\mathbb{P}^{1}\right)^{4}\right)$ as

$(22)\left(\left[\mathbb{P}^{1} \times 0\right]+\left[0 \times \mathbb{P}^{1}\right]+\left(t_{1}-t_{2}\right)\left[\mathbb{P}^{1} \times \mathbb{P}^{1}\right]\right) \times\left(\left[\mathbb{P}^{1} \times 0\right]+\left[0 \times \mathbb{P}^{1}\right]+\left(t_{1}-t_{2}\right)\left[\mathbb{P}^{1} \times \mathbb{P}^{1}\right]\right)$.

When $k \geq 2$ we substitute (22) and use the permutation invariance of $\rho_{*}$ to obtain

$$
\begin{aligned}
\rho_{*}\left[\left(0 \times \mathbb{P}^{1}\right)^{k}\right. & \left.\times\left(\Delta_{\mathbb{P}^{1} \times \mathbb{P}^{1}}\right)^{2} \times\left(\mathbb{P}^{1}\right)^{N-2(k+2)}\right]=(N-k) !\left(t_{1}-t_{2}\right)^{2} h_{0} \ldots h_{k-1} \\
& +4(N-(k+1)) !\left(t_{1}-t_{2}\right) h_{0} \ldots h_{k}+4(N-(k+2)) ! h_{0} \ldots h_{k+1}
\end{aligned}
$$

When $k=1$ we use the same expansion to obtain that

$$
\begin{aligned}
\rho_{*}\left(\left[0 \times \mathbb{P}^{1} \times\left(\Delta_{\mathbb{P}^{1} \times \mathbb{P}^{1}}\right)^{2} \times\left(\mathbb{P}^{1}\right)^{N-6}\right]\right)=4(N-3) ! h_{0} h_{1} h_{2} \\
\quad+4(N-2) !\left(t_{1}-t_{2}\right) h_{0} h_{1}+(N-1) !\left(t_{1}-t_{2}\right)^{2} h_{0}
\end{aligned}
$$

Since $r \geq 3$, the first term on the right-hand-side of $(21)$ is divisible $r !(N-2 r) ! \alpha_{2,0}$ and thus is an element of $r !(N-2 r) !\left(\alpha_{1,0}, h_{0} h_{1}\right)$ by Lemma 4.7. Likewise, each of the three terms on the right-hand-side of $(23)$ is divisible $r !(N-2 r) ! h_{0} h_{1}$ as are the first 
two terms on the right-hand-side of (24). Finally by (19) we know that $(N-1)(N-$ $2)\left(t_{1}-t_{2}\right) h_{0} \in\left(\alpha_{1,0}, h_{0} h_{1}\right)$. Hence $(N-1) !\left(t_{1}-t_{2}\right) h_{0}$ is in $(N-3) !\left(\alpha_{1,0}, h_{0} h_{1}\right)$. Since $r \geq 3, r !(N-2 r) ! \mid(N-3) !$, so $(N-1) !\left(t_{1}-t_{2}\right) h_{0}$ is in $r !(N-2 r) !\left(\alpha_{1,0}, h_{0} h_{1}\right)$. Hence all of the terms appearing on the right hand side of (24) are in $r !(N-2 r) !\left(\alpha_{1,0}, h_{0} h_{1}\right)$. Since $A_{T}^{*}\left(\mathbb{P}^{N}\right)$ is torsion free we conclude that $\alpha_{r, 0} \in\left(\alpha_{1,0}, h_{0} h_{1}\right)$.

4.2. The ideal generated by the image of $A_{*}^{\mathrm{GL}_{2}}\left(\Delta_{1}\right)$ in $A_{\mathrm{GL}_{2}}^{*}\left(\mathbb{P}^{N}\right)$. We are now in a position to prove our main result

Theorem 4.9. If char $k=0$ or char $k>N$ then the image of $A_{*}^{\mathrm{GL}_{2}}\left(\Delta_{1}\right)$ in $A_{\mathrm{GL}_{2}}^{*}\left(\mathbb{P}^{N}\right)$ is the ideal $I=\left(\alpha_{1,0}, \alpha_{1,1}\right)$.

Remark 4.10. When $N=6$ this result was previously obtained by Vistoli [14] for his calculation of $A^{*} \mathcal{M}_{2}$.

Proof. Since $\mathrm{GL}_{2}$ is special, $A_{\mathrm{GL}_{2}}^{*}\left(\mathbb{P}^{N}\right)$ is (non-canonically) a summand in the $A_{\mathrm{GL}_{2}}^{*}\left(\mathbb{P}^{N}\right)$-module $A_{T}^{*}\left(\mathbb{P}^{N}\right)$ by Proposition 2.2. Thus we may decompose the $A_{\mathrm{GL}_{2}}^{*}\left(\mathbb{P}^{N}\right)$-module $A_{T}^{*}\left(\mathbb{P}^{N}\right)$ as $A_{T}^{*}\left(\mathbb{P}^{N}\right)=A_{\mathrm{GL}_{2}}^{*}\left(\mathbb{P}^{N}\right) \oplus M$ where $M \subset A_{T}^{*}\left(\mathbb{P}^{N}\right)$ is a complimentary submodule.

Now suppose that $f \in A_{\mathrm{GL}_{2}}^{*}\left(\mathbb{P}^{N}\right)$ is in the image of $A_{*}^{\mathrm{GL}_{2}}\left(\Delta_{1}\right)$. Since the inclusion of $A_{\mathrm{GL}_{2}}^{*}\left(\Delta_{1}\right)$ in $A_{T}^{*}\left(\Delta_{1}\right)$ commutes with the direct image map $i_{*}: \Delta_{1} \rightarrow \mathbb{P}^{N}$, we may view $f$ as being in the image of $A_{T}^{*}\left(\Delta_{1}\right)$. Thus by our previous proposition we may write $f=a \alpha_{1,0}+b \alpha_{1,1}$ for some $a, b \in A_{T}^{*}\left(\mathbb{P}^{N}\right)$. Using the decomposition above we may write $a=a_{s}+a_{u}, b=b_{s}+b_{u}$ with $a_{s}, b_{s}$ in $A_{\mathrm{GL}_{2}}^{*}\left(\mathbb{P}^{N}\right)$ and $a_{u}, b_{u}$ in $M$. Thus

$$
f=a_{s} \alpha_{1,0}+b_{s} \alpha_{1,1}+a_{u} \alpha_{1,0}+b_{u} \alpha_{1,1} .
$$

Since $f \in A_{\mathrm{GL}_{2}}^{*}\left(\mathbb{P}^{N}\right)$ it follows that $a_{u} \alpha_{1,0}+b_{u} \alpha_{1,1}$ is an element of $M \cap A_{\mathrm{GL}_{2}}^{*}\left(\mathbb{P}^{N}\right)=$ $\{0\}$. Hence $f=a_{s} \alpha_{1,0}+b_{s} \alpha_{1,1}$, so $f$ is in the ideal of $A_{\mathrm{GL}_{2}}^{*}\left(\mathbb{P}^{N}\right)$ generated by $\alpha_{1,0}$ and $\alpha_{1,1}$.

\section{Proof of Theorem 1.1}

We can now easily complete the proof of Theorem 1.1. By Theorem 4.9

$$
A_{\mathrm{GL}_{2}}^{*}\left(\mathbb{P}^{2 g+2}-\Delta_{1}\right)=A_{\mathrm{GL}_{2}}^{*}\left(\mathbb{P}^{2 g+2}\right) /\left(\alpha_{1,0}, \alpha_{1,1}\right)
$$

where

$$
\begin{aligned}
& \alpha_{1,0}=2(2 g+1) \xi-(2 g+2)(2 g+1) c_{1} \\
& \alpha_{1,1}=\xi^{2}-\xi_{1} c_{1}-(2 g+2)(2 g) c_{2}
\end{aligned}
$$

If $C_{1}, \ldots, C_{2 g+3}$ are the $\mathrm{GL}_{2}$-equivariant Chern classes of the representation $\mathrm{Sym}^{2 g+2} E^{*}$ set

$$
P=\xi^{2 g+3}+C_{1} \xi^{2 g+1}+\ldots+C_{2 g+2}
$$

so that $A_{\mathrm{GL}_{2}}^{*}\left(\mathbb{P}^{2 g+2}\right)=\mathbb{Z}\left[c_{1}, c_{2}\right][\xi] / P$ by Lemma 2.3 . Let $\alpha_{1,0}\left(g c_{1}\right), \alpha_{1,0}\left(g c_{1}\right), P\left(g c_{1}\right)$ be the polynomials in $c_{1}, c_{2}$ obtained by substituting $\xi=g c_{1}$ in (25)-(27) then by Lemma 3.2,

$$
A^{*}\left(\mathcal{H}_{g}\right)=\mathbb{Z}\left[c_{1}, c_{2}\right] /\left(\alpha_{1,0}\left(g c_{1}\right), \alpha_{1,1}\left(g c_{1}\right), P\left(g c_{1}\right)\right)
$$


where

$$
\begin{aligned}
& \alpha_{1,0}\left(g c_{1}\right)=-2(2 g+1) c_{1} \\
& \alpha_{1,1}\left(g c_{1}\right)=-g(g-1) c_{1}^{2}+4 g(g+1) c_{2}
\end{aligned}
$$

The theorem then follows from our last lemma.

Lemma 5.1. The polynomial $P\left(g c_{1}\right)$ is in the ideal $\left(\alpha_{1,0}\left(g c_{1}\right), \alpha_{1,1}\left(g c_{1}\right)\right)$.

Proof. Using the same arguments as in Section 4.2 it suffices to show that the restriction of $P\left(g c_{1}\right)$ to $A_{T}^{*}$ is in the ideal generated by the restrictions of $\alpha_{1,0}\left(g c_{1}\right)$ and $\alpha_{1,1}\left(g c_{1}\right)$.

The Chern roots of $E$ are

$$
\left\{-(2 g+2) t_{1},-(2 g+1) t_{1}-t_{2}, \ldots,-t_{1}-(2 g+1) t_{2},-(2 g+2) t_{2}\right\}
$$

so

$$
P\left(g c_{1}\right)=\prod_{i=0}^{2 g+2}\left(g c_{1}-(2 g+2-i) t_{1}-i t_{2}\right)
$$

Pairing off the $2 g$ terms $\left(g c_{1}-(2 g+2-i) t_{1}-i t_{2}\right)$ and $\left(g c_{1}-i t_{1}-(2 g+2-i) t_{2}\right)$ and observing that if $i=g+1$ then $g c_{1}-(2 g+2-i) t_{1}-i t_{2}=-c_{1}$ we can rewrite $(28)$ as

$$
P\left(g c_{1}\right)=-c_{1} \prod_{j=0}^{g}\left(-(g-j)(g-j+2) c_{1}^{2}+4(g+1-j)^{2} c_{2}\right)
$$

The product of the $j=0$ and $j=1$ terms on the right hand side of (29) is

$$
\begin{aligned}
Q\left(c_{1}, c_{2}\right) & =\left((g-1)(g+1) c_{1}^{2}-4 g^{2} c_{2}\right)\left(g(g+2) c_{1}^{2}-4(g+1)^{2} c_{2}\right) \\
& =\left(\alpha_{1,0}\left(g c_{1}\right)\right)^{2} c_{2}+\alpha_{1,0}\left(g c_{1}\right) \alpha_{1,1}\left(g c_{1}\right) c_{1}+\left(\alpha_{1,1}\left(g c_{1}\right)\right)^{2}
\end{aligned}
$$

5.1. Tautological classes. The identification, for $g$ even, of

$$
\mathcal{H}_{g}=\left[\left(\mathbb{A}^{2 g+3} \backslash \Delta_{1}\right) / \mathrm{GL}_{2}\right]
$$

means that the defining representation of $\mathrm{GL}_{2}$ determines a vector bundle on $\mathcal{H}_{g}$ whose Chern classes generate the Chow ring. Using an observation of Gorchinskiy and Viviani we can obtain a functorial geometric description of this bundle.

If $\pi: X \rightarrow S$ is a family of smooth hyperelliptic curves of genus $g$ let $W \subset X$ be the divisor of Weierstrass points of the fibers of $\pi$ and let $\omega$ be the relative canonical line bundle. Then $\mathcal{O}_{X}(W)$ has relative degree $2 g+2$ and the line bundle $\omega_{\pi}^{\otimes g / 2} \otimes$ $\mathcal{O}_{X}((1-g / 2) W)$ restricts to a $g_{2}^{1}$ on the fibers of $\pi$. Since $\pi$ is flat, it follows that

$$
V_{\pi}=\pi_{*}\left(\omega_{\pi}^{\otimes g} / 2 \otimes \mathcal{O}_{X}((1-g / 2) W)\right.
$$

is rank 2 vector bundle on $S$. Let $\mathcal{V}_{g}$ be the rank two bundle on the stack $\mathcal{H}_{g}$ which restricts to to $V_{\pi}$ on a family of hyperelliptic curves $X \stackrel{\pi}{\rightarrow} S$.

Proposition 5.2. Under the identification $\mathcal{H}_{g}=\left[\left(\mathbb{A}^{2 g+3} \backslash \Delta_{1}\right) / \mathrm{GL}_{2}\right]$ the vector bundle $\mathcal{V}_{g}$ corresponds to the defining representation of $\mathrm{GL}_{2}$. In particular the Chow ring of $\mathcal{H}_{g}$ is generated by the Chern classes of $\mathcal{V}_{g}$. 
Remark 5.3. When $g=2$, then $\mathcal{V}_{g}$ is the Hodge bundle and we recover Vistoli's result [14] that $A^{*}\left(\mathcal{M}_{2}\right)$ is generated by the Chern classes of the Hodge bundle.

Remark 5.4. As observed in [10] the Chern classes of $\mathcal{V}_{g}$ are not tautological classes. Gorchinskiy and Viviani show that the first Hodge class $\lambda$ equals $(g / 2) c_{1}$. In principal the methods of their paper could be extended to give formulas for all of the tautological classes in terms of $c_{1}$ and $c_{2}$, but we do not pursue this here.

Proof. Following [2] and [10] we know that given a family of hyperelliptic curves $\pi: X \rightarrow S$ the map $\pi$ factors as $\pi=p \circ f$ where $p: P \rightarrow S$ is a Brauer-Severi variety and $f: X \rightarrow P$ is a double cover. Then $f_{*} \mathcal{O}_{X}=O_{P} \oplus \mathcal{L}$ where $\mathcal{L}$ is a line-bundle such that $\mathcal{L}^{2}=\mathcal{O}_{P}(-D)$ where $D \subset P$ is the ramification divisor. Since $f$ is a double cover, the family of elliptic curves is uniquely determined by the data $(p: P \rightarrow S, \mathcal{L})$.

The identity $f^{*} \mathcal{L}^{-1}=\mathcal{O}_{X}(W)$ and the Riemann-Hurwitz formula imply that

$$
f_{*}\left(\omega^{\otimes g / 2} \otimes \mathcal{O}_{X}((1-g / 2) W)=\omega_{P / S}{ }^{\otimes g / 2} \otimes \mathcal{L}^{-1} \otimes\left(\mathcal{O}_{P} \oplus \mathcal{L}\right)\right.
$$

Since the restriction of $\omega_{P / S}^{\otimes g / 2}$ has degree $-g$ we see that

$$
\pi_{*}\left(\omega^{\otimes g / 2} \otimes \mathcal{O}_{X}((1-g / 2) W)=p_{*}\left(\omega_{P / S}^{\otimes g / 2} \otimes \mathcal{L}^{-1}\right) .\right.
$$

As noted in [2, Remark 3.3] we may identify $\mathcal{H}_{g}$ with the stack $\mathcal{H}_{1,2, g+1}^{\prime}$ whose objects over a $k$-scheme $S$ consists of the data of a Brauer-Severi variety $p: P \rightarrow S$ together with a line bundle $\mathcal{L}$ of relative degree $-(g+1)$ and an injection $i: \mathcal{L}^{2} \hookrightarrow \mathcal{O}_{P}$. From the previous paragraph we see that we may identify $\mathcal{V}_{g}$ with the bundle whose restriction to $P \rightarrow S$ is the vector bundle $p_{*}\left(\omega_{P / S}^{\otimes g / 2} \otimes \mathcal{L}^{-1}\right)$.

Let $\tilde{\mathcal{H}}_{1,2, g+1}$ be the stack whose objects over $S$ consists of a pair $(P \stackrel{p}{\rightarrow} S, \mathcal{L})$ together with an isomorphism $\phi:(P, \mathcal{L}) \rightarrow\left(\mathbb{P}_{S}^{1}, \mathcal{O}_{\mathbb{P}^{1}}(-g-1)\right)$. The action of $\mathrm{GL}_{2}:=$ $\operatorname{Aut}\left(\mathbb{P}^{1}, \mathcal{O}(1)\right)$ on the pair $\left(\mathbb{P}^{1}, \mathcal{O}(-g-1)\right)$ has kernel $\mu_{g-1}$ and by $[2$, Theorem 4.1] $\tilde{\mathcal{H}}_{1,2, g+1}$ is represented by the scheme $\mathbb{A}^{2 g+3} \backslash \Delta_{1}$ and the forgetful map $\tilde{\mathcal{H}}_{1,2, g+1} \rightarrow$ $\mathcal{H}_{1,2, g+1}$ is a $\mathrm{GL}_{2} / \mu_{g+1}$-torsor.

The vector bundle $\mathcal{V}_{g}$ pulls back to the vector bundle which assigns to the trivial family $p_{*}: \mathbb{P}_{S}^{1} \rightarrow S$ the vector bundle $p_{*}\left(\omega_{\mathbb{P}^{1}}^{\otimes g / 2} \otimes \mathcal{O}_{\mathbb{P}^{1}}(g+1)\right)$. As noted in [10, Equation (4.4)] the Euler sequence for the tangent bundle of $\mathbb{P}^{1}$ implies $\omega_{\mathbb{P}^{1}}$ is $\mathrm{GL}_{2}$-equivariantly isomorphic to the bundle $\mathcal{O}(-2) \otimes \operatorname{det} E$ where $E$ is the defining representation of $\mathrm{GL}_{2}$. Thus,

$$
p_{*}\left(\omega_{\mathbb{P}^{1}}^{\otimes g / 2} \otimes \mathcal{O}_{\mathbb{P}^{1}}(g+1)\right)=(\operatorname{det} E)^{\otimes g / 2} \otimes E .
$$

Now $(\operatorname{det} E)^{\otimes g / 2} \otimes E$ is the pullback of $E$ via the map $\alpha: \mathrm{GL}_{2} \rightarrow \mathrm{GL}_{2}, A \mapsto$ $(\operatorname{det} A)^{g / 2} A$. As noted in [2] $\operatorname{ker} \alpha=\mu_{g+1}$, so under the identification of

$$
\mathrm{GL}_{2} / \mu_{g+1}=\mathrm{GL}_{2},
$$

$(\operatorname{det} E)^{\otimes g / 2} \otimes E$ corresponds to the defining representation $E$.

\section{Acknowledgements}

The authors are grateful to Fillipo Viviani for pointing out that our proof works under the assumption that char $k=0$ or char $k>2 g+1$ rather than char $k>2 g$ as we originally stated. The reason for this increase in characteristic is that Proposition 4.1 
requires that char $k=0$ or char $k>N$, rather than char $k>N-2$ as we originally claimed.

The authors are also grateful to the referee for a careful reading.

\section{References}

[1] E. Arbarello and M. Cornalba, The Picard groups of the moduli spaces of curves, Topology 26 (2004), no. 2, 153-171.

[2] A. Arsie and A. Vistoli, Stacks of cyclic covers of projective spaces, Compos. Math. 140 (2004), no. $3,647-666$.

[3] M. Brion, Equivariant Chow groups for torus actions, Transform. Groups 2 (1997), no. 3, 225267.

[4] F. R. Cohen and M. D. Peim, Mapping class groups and function spaces, in Homotopy methods in algebraic topology (Boulder, CO, 1999), Vol. 271, 17-39, Contemp. Math., Providence, RI (2001).

[5] D. Edidin and W. Graham, Characteristic classes in the Chow ring, J. Algebraic Geom. 6 (1997) 431-443.

[6] - Equivariant intersection theory, Invent. Math. 131 (1998), no. 3, 595-634.

[7] C. Faber, Chow rings of moduli spaces of curves I. The Chow ring of $\overline{\mathcal{M}}_{3}$, Ann. of Math. (2) 132 (1990), no. 2, 331-419.

[8] Chow rings of moduli spaces of curves II. Some results on the Chow ring of $\overline{\mathcal{M}}_{4}$, Ann. of Math. (2) 132 (1990), no. 3, 421-449.

[9] W. Fulton, Intersection theory, Springer-Verlag, Berlin (1984).

[10] S. Gorchinskiy and F. Viviani, Picard group of moduli of hyperelliptic curves, Math. Z. 258 (2008), no. 2, 319-331.

[11] J. Harer, The second homology group of the mapping class group of an orientable surface, Invent. Math. 72 (1983), no. 2, 267-304.

[12] E. Izadi, The Chow ring of the moduli space of curves of genus 5, in P. Math., editor, The moduli space of curves (Texel Island, 1994), Vol. 129, 267-304, Birkhäuser Boston, Boston, MA (1995).

[13] D. Mumford, Towards an enumerative geometry of the moduli space of curves, in P. Math., editor, Arithmetic and geometry, Vol. II, Vol. 36, 271-328, Birkhäuser Boston, Boston, MA (1983).

[14] A. Vistoli, The Chow ring of $\mathcal{M}_{2}$, Invent. Math. 131 (1998), no. 3, 635-644.

Department of Mathematics, University of Missouri, Columbia, MO 65211

E-mail address: edidin@math.missouri.edu, damiano@math.missouri.edu 